\title{
De la concertation territoriale à l'expérimentation en plein champs, différents leviers pour accompagner les acteurs d'un territoire agricole à façonner des paysages durablement favorables à des productions oléagineuses et des productions de miel
}

\author{
Marine Gourrat ${ }^{*}$ \\ ITSAP-Institut de l'abeille, 149 rue de Bercy, 75595 Paris cedex 12, France
}

Reçu le 6 juillet 2017 - Accepté le 29 août 2017

\begin{abstract}
Résumé - Les interactions entre les abeilles et leur milieu sont multiples, complexes, incomprises pour certaines et parfois même controversées. Cette complexité se répercute parfois dans les échanges entre les acteurs liés de près ou de loin à ces insectes pollinisateurs qui évoluent sur un même territoire. Dans ce contexte, l'institut technique et scientifique de l'apiculture et de la pollinisation (ITSAP-Institut de l'abeille), après avoir fait le constat qu'apiculteurs et agriculteurs ne partagent pas toujours la même perception de leur territoire, travaille depuis quelques années à accompagner ces acteurs dans la construction de leviers favorables au maintien durable de ces différentes activités agricoles sur leur territoire. Nous illustrerons cette dynamique à travers différents formats d'actions : l'échange de connaissances dans des ateliers de concertation sous forme de jeu de rôles et l'acquisition et le partage de références dans une expérimentation en plein champs.
\end{abstract}

\begin{abstract}
Mots clés : système de culture / abeilles / accompagnement d'acteurs / ateliers de concertation / expérimentation en plein champs

Abstract - From collaborative workshops to field experiment, some ways to support agricultural stakeholders in building sustainable landscapes for both oilseed crops and honey production. Interactions between bees and their environment are various and complex. They are also misunderstood and sometimes controversial. This complexity can affect the relationships between stakeholders who are closely or indirectly concerned with pollinating insects and who work in the same territory. In this context, French technical and scientific institute of beekeeping and pollination (ITSAP), has observed that beekeepers and farmers do not always share the same perception of their activity. The institute works now to support them in producing bee-friendly alternatives. The approach is based on helping stakeholders share a common objective: sustainably maintain a diversity of production (crop and honey) on their land. This approach will be illustrated through different actions: sharing knowledge thanks to collaborative workshops and collecting data in a field experiment that can be shared...
\end{abstract}

Keywords: cropping system / bees / stakeholders engagement / collaborative workshops / field experiment

\section{Introduction}

Les productions oléagineuses et la production de miel sont dans beaucoup de régions de France fortement interdépendantes: location de ruches par des multiplicateurs de semences de colza ou de tournesol pour assurer une production de graines; production de miels de colza et de tournesol qui peut représenter

${ }^{*}$ Correspondance : marine.gourrat@itsap.asso.fr une part majeur du volume de miel produit annuellement au sein d'une exploitation [jusqu'à $87 \%$ dans la région Poitou-Charente en 2004 (Gem-Oniflhor, 2005)]. Ces deux activités souffrent aujourd'hui du déclin des abeilles domestiques (Potts et al., 2010). Le maintien durable de cette pluriactivité au sein des territoires dépend donc en partie de l'adoption de pratiques agricoles et apicoles favorables aux enjeux de ces différentes productions. Pourtant, de nombreuses interactions qui lient ces activités restent soumises à controverse [impact des pesticides sur la dynamique d'une colonie (Maxim et Sluijs, 

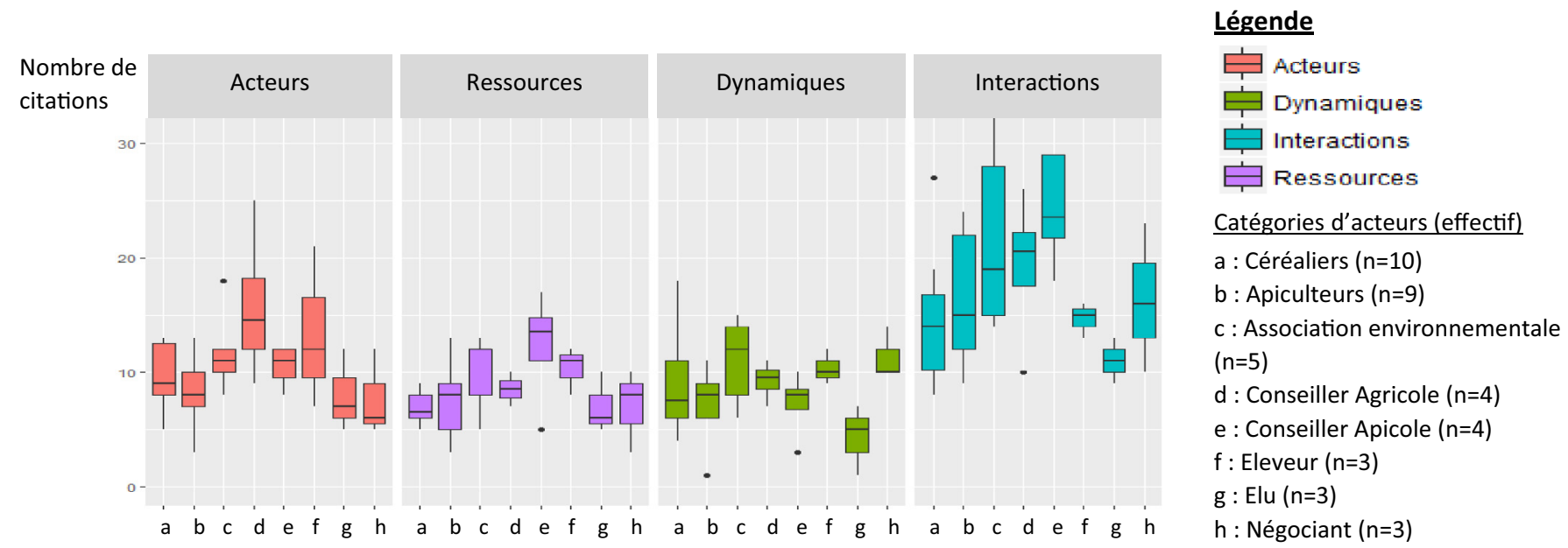

Fig. 1. Nombre d'acteurs, de ressources, de dynamiques et d'interactions différentes cités par chacune des catégories d'acteurs interrogés, concernés selon eux par l'utilisation des terres cultivées pour produire en zone de grandes cultures.

2013 ; Suryanarayanan et Kleinman, 2013; Cressey, 2013), apport de la pollinisation par les abeilles sur le rendement des cultures de rentes (Bommarco et al., 2012; Stanley et al., 2013)], ce qui peut cristalliser voire dégrader le dialogue entre exploitants sur le terrain. L'évolution des territoires vers une coexistence durable des productions oléagineuses et de miel nécessite donc à la fois l'acquisition de nouvelles références via l'expérimentation et la recherche mais également l'appropriation des différents enjeux du territoire par l'ensemble des acteurs concernés. C'est l'objectif qui anime les travaux de l'institut technique et scientifique de l'apiculture et de la pollinisation (l'ITSAP-Institut de l'abeille), menés en partenariat avec les acteurs des différentes filières, dans le but d'initier à travers une concertation territoriale, la conception, l'expérimentation et l'adoption de systèmes de cultures favorables aux insectes pollinisateurs. Nous illustrerons dans cet article comment à travers l'échange de connaissances au sein d'ateliers de concertations ou l'acquisition de nouvelles références au sein d'une expérimentation de plein champs, l'ITSAP-Institut de l'abeille accompagne les acteurs agricoles à faire émerger collectivement et localement des idées d'actions à entreprendre.

\section{La concertation entre producteurs d'oléagineux et apiculteurs, une étape vers l'évolution des systèmes de cultures sur le territoire}

\subsection{Apiculteurs et cultivateurs évoluent sur un même territoire qu'ils perçoivent différemment}

En zone cultivée, l'existence d'interactions entre l'abeille mellifère et les champs cultivés est évidente et complexe: l'agriculteur est perçu à la fois comme producteur de ressources alimentaires pour les insectes pollinisateurs pouvant conduire à une miellée et comme un acteur impliqué dans l'affaiblissement de ces insectes ; mais cette complexitén'est pas toujours partagée par l'ensemble des acteurs concernés par ces interactions. C'est ce qu'a démontré une série d'entretiens menés auprès de plus d'une trentaine de personnes revendiquant des savoirs sur l'abeille mellifère et/ou l'agriculture (apiculteurs, céréaliers, élus, associations environnementales, techniciens de coopératives...). Ces enquêtes conduites au sein de deux territoires de grandes cultures: la région centre (projet InterAPI CASDAR 2011-2013) et la plaine de Niort (projet DEPHY-Abeille DEPHY EXPE 2013-2018) ont consisté à faire expliciter les Acteurs, les Ressources, les Dynamiques et les Interactions concernés selon chaque personne interrogée par l'utilisation des terres cultivées sur leur territoire (méthode ARDI, Etienne et al., 2011). Leur objectif était de connaître la vision de ces différents acteurs du territoire sur les productions agricoles (Allier et al., 2017). Le nombre d'acteurs, de ressources, de dynamiques et d'interactions cités est très variable au sein d'une même catégorie d'acteurs (Fig. 1). Par ailleurs, bien que la complexité des représentations conceptuelles ne diffère pas significativement entre elles, les personnes rencontrées ne donnent qu'une vision partielle de la question posée, bien souvent cloisonnée à leur propre activité (Fig. 2 et 3). L'apiculteur ou l'abeille sont par exemple souvent absents de la représentation que les agriculteurs ont d'un paysage agricole productif. L'étude met par ailleurs en évidence des visions parfois opposées entre ces deux professions sur l'action de certaines ressources (intrants $=$ stimulateurs versus toxiques) ou disjointes (produit de la plante $=$ grain versus nectar), ainsi que des méconnaissances réciproques ou des incertitudes. Ces différents éléments mettent en avant l'importance de mettre en commun les savoirs et points de vue des acteurs d'un territoire, d'une part pour élargir et complexifier leur perception de leur environnement et d'autre part pour discuter des points clés où une compréhension commune entre eux fait défaut.

\subsection{La concertation comme source de changements techniques favorable à une pluriactivité apicole et cultures oléagineuses sur le territoire}

\subsubsection{Un outil de concertation éprouvé dans différents contextes de productions}

Partant du constat qu'apiculteurs et agriculteurs d'un même territoire n'ont pas la même perception de leur environnement et faisant l'hypothèse qu'une vision commune peut émerger en collectif, l'ITSAP a entamé une démarche de modélisation d'accompagnement (Daré et al., 2009; Barreteau et al., 2010). 


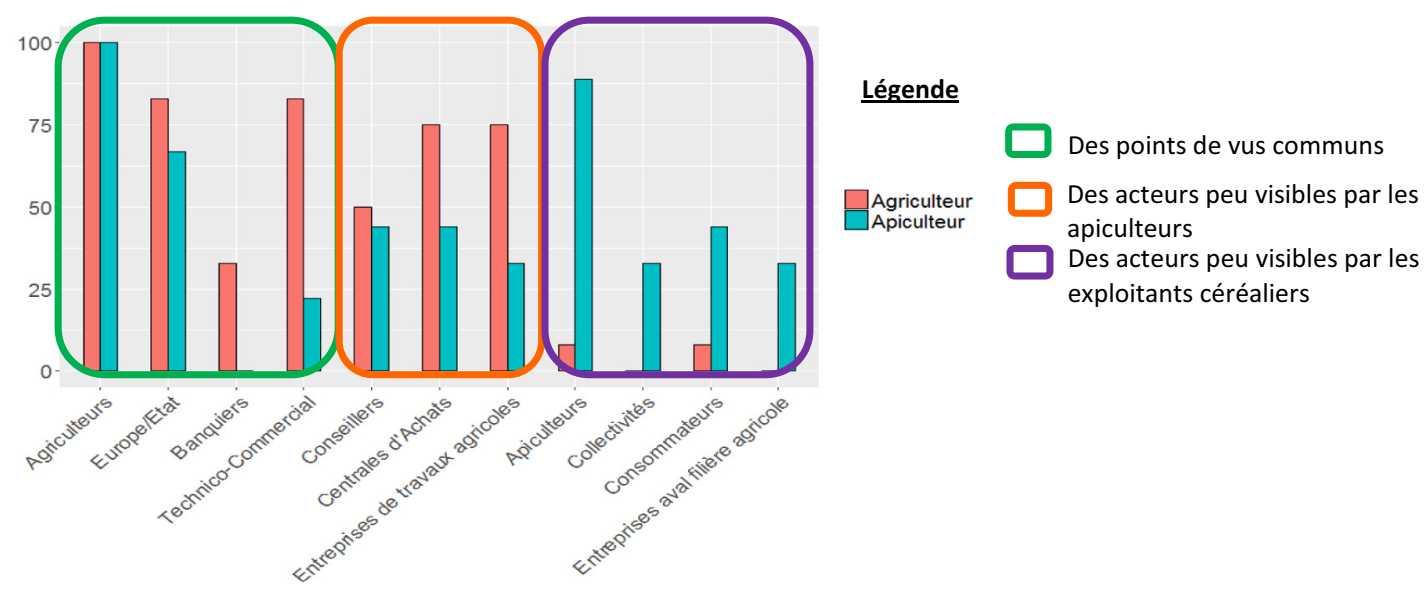

Fig. 2. Pourcentage d'agriculteurs et d'apiculteurs interviewés qui cite chacun des acteurs, concernés selon eux par la question de l'utilisation des terres cultivées sur leur territoire.

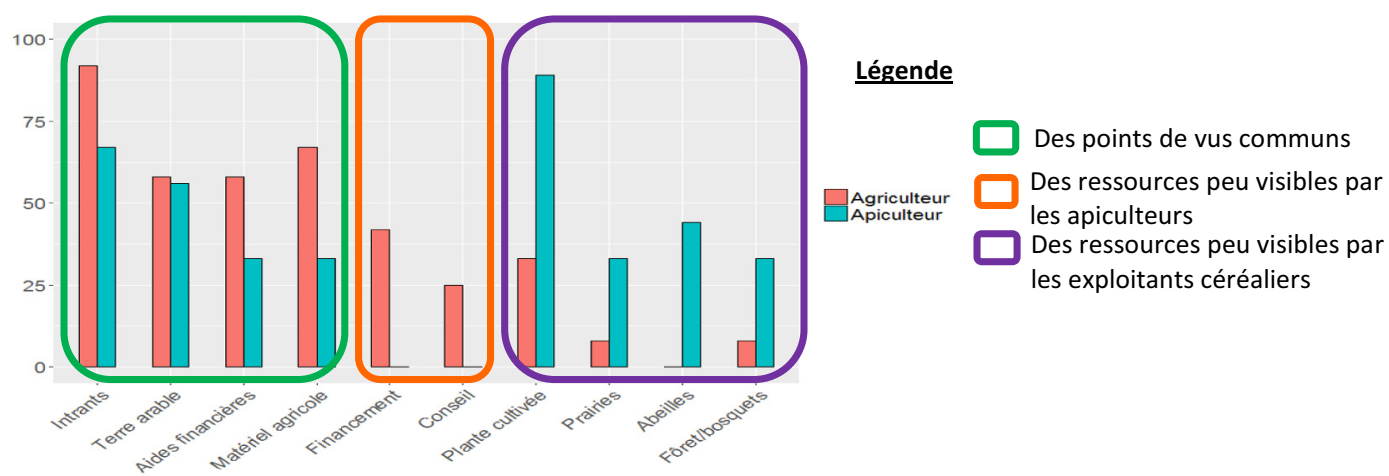

Fig. 3. Pourcentage d'agriculteurs et d'apiculteurs interviewés qui cite chacune des ressources, concernées selon eux par la question de l'utilisation des terres cultivées sur leur territoire.

Un format original d'atelier d'échanges reposant sur la mise en situation sous forme d'un jeu de rôles a ainsi été créé (Allier et al., 2017). Depuis l'année 2014, l'ITSAP-Institut de l'abeille a animé 13 ateliers de concertation sous ce format original adapté à chaque territoire, dans différents bassins de production de culture oléagineuses (soit dans le cadre de projet de recherche en Plaine de Niort, soit suite à la sollicitation d'une coopérative agricole en région Poitou-Charente, soit dans le cadre de la Démarche de Progrès Colza Diester ${ }^{\circledR}$, en partenariat avec le groupe Avril et la fédération française des producteurs d'oléagineux et de protéagineux (FOP) en Bourgogne, Champagne Ardenne, Haut de France et région Centre). Ces derniers ont rassemblé apiculteurs, cultivateurs, éleveurs et représentants de coopératives, du même territoire.

À chacune des mises en situation, une a plusieurs années culturales sont simulées. Les exploitants présents façonnent à travers leurs choix d'assolement, un paysage sur lequel il est demandé aux apiculteurs de faire du miel. Les éléments semisnaturels ne sont volontairement pas figurés sur le territoire afin de mettre en avant les liens entre les deux systèmes de production et forcer les interactions pendant la mise en situation. Les ressources alimentaires et les ressources mellifères disponibles sont ainsi directement influencées par le choix des exploitants qui participent.

Lors de la mise en situation, les exploitants céréaliers et apiculteurs rendent compte également de la gestion habituelle de leurs ruches ou de leurs cultures. Il est par exemple demandé aux exploitants céréaliers de tenir un cahier de plaine et d'y indiquer le nombre de passages de traitements phytosanitaires. Les territoires et pratiques simulées (Fig. 4 et 5) démontrent une bonne appropriation de la mise en situation par les participants. La variabilité observée illustre par ailleurs la diversité des bassins de production où ont été animés les ateliers.

Les échanges au cours de l'atelier contribuent dans un premier temps à faire évoluer la perception de chacun sur l'activité voisine. L'animation de l'atelier fait ressortir les similitudes entre la conduite d'une exploitation céréalière et celle d'une exploitation apicole. Comme cela a été, à de nombreuses reprises exprimé par les participants en fin d'atelier, tous sont des exploitants agricoles dont l'activité repose sur une production (grain ou miel) et dont les choix techniques sont raisonnés en fonction de différents enjeux (économiques, sociaux ou environnementaux). Le format de l'atelier permet de discuter à partir d'un socle commun (une mise en situation fictive d'une ou plusieurs années culturales sous forme de jeu) ce qui permet de dépassionner certains sujets de discussions qui par ailleurs peuvent être conflictuels. Les thématiques abordées sont ainsi pour la plupart traitées sous un angle technique (règles de décisions qui encadrent un traitement phytosanitaires; troubles observés sur des colonies en pleine saison...). Le collectif fait alors parfois le constat que certaines controverses au sujet des interactions entre leurs deux activités ont pour origine des trous 


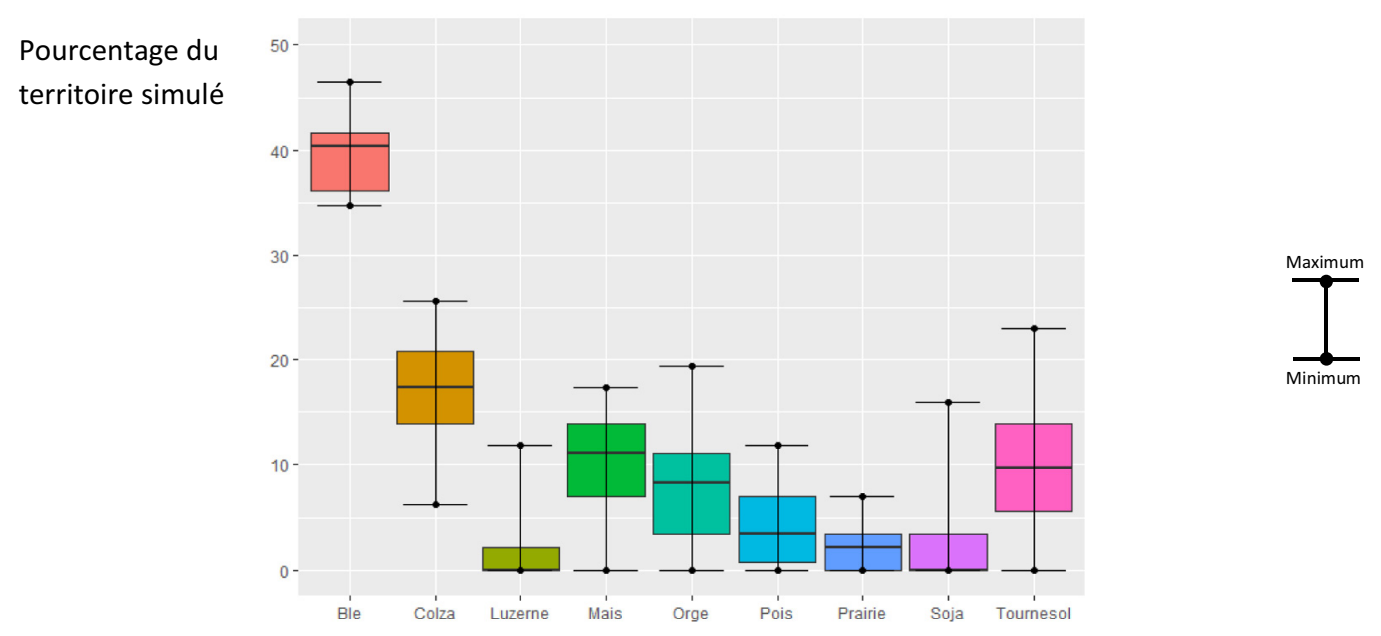

Fig. 4. Distribution, maximum et minimum des proportions des principales cultures dans les territoires simulés lors des 13 sessions d'ateliers de mise en situation animés dans différentes régions françaises.

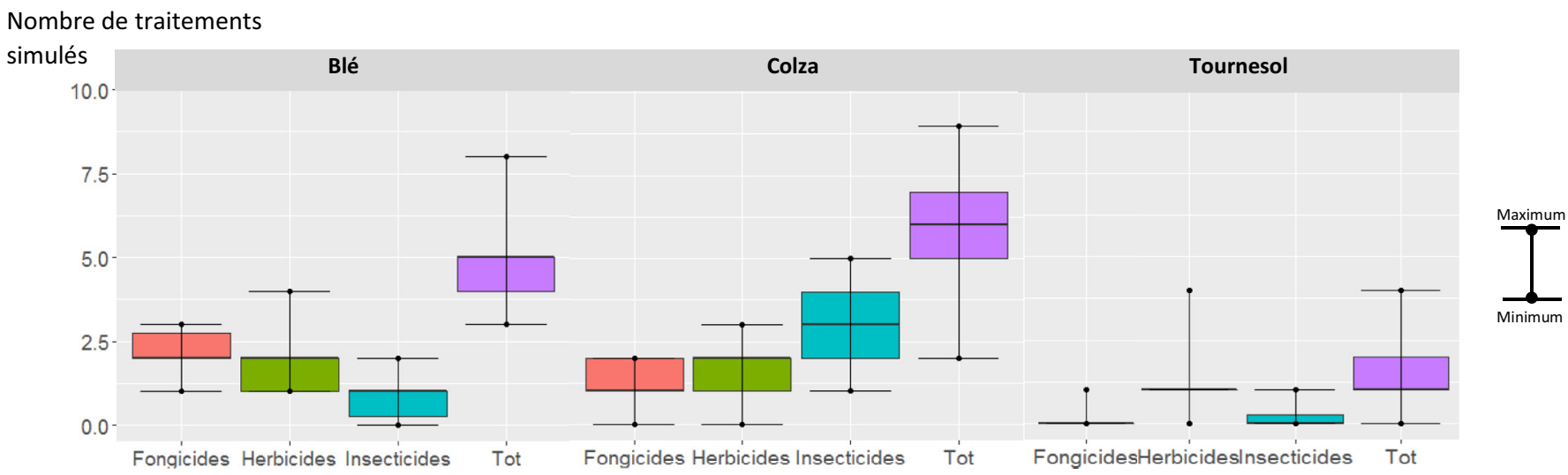

Fig. 5. Distribution, maximum et minimum du nombre de traitements fongicides, herbicides et insecticides sur les 3 principales cultures des assolements (blé, colza et tournesol) simulés lors des 13 sessions d'ateliers de mise en situation animés dans différentes régions françaises.

de connaissances (contribution des abeilles domestiques au rendement des cultures par exemple). Les 13 ateliers organisés ont par ailleurs mis en avant qu'apiculteurs et exploitants céréaliers se connaissent peu. Ils évoquent eux-mêmes «deux mondes hermétiques ». Une part importante des ateliers est ainsi dédiée à l'enrichissement des connaissances de chacun sur les différentes activités.

\subsubsection{La concertation, une source de pistes d'actions répondant à des enjeux multiples}

En plus de favoriser l'interconnaissance, l'atelier invite les participants à réfléchir collectivement à des leviers d'actions pour favoriser le maintien durable de leurs activités sur leur territoire. Ces pistes reposent à la fois sur des savoirs techniques, des savoirs scientifiques et du lien social.

\subsubsection{Les échanges entre filières: un besoin exprimé dans chacun des ateliers}

La connaissance de « l'autre », a été reconnue par l'ensemble des participants comme un atout pour aller vers une évolution des pratiques au sein des territoires. Un exploitant a notamment souligné que « le changement de pratique ne se faisait pas dans l'opposition mais dans le but de servir une cause ou un objectif ». Une réflexion sur les modalités et formats d'échanges pouvant favoriser ces échanges a ainsi été menée au sein de chaque atelier. Différentes pistes pour renouer le dialogue entre professionnels ont ainsi été évoquées : information technique à destination des conseillers, lieux de rencontres par l'intermédiaire des communes, des coopératives...

\subsubsection{Des pistes techniques discutées à différentes échelles (parcelle/exploitation, paysage...)}

Les participants se sont également constamment questionnés sur ce qu'était un paysage agricole favorable aux insectes pollinisateurs et comment une exploitation agricole pouvaient y contribuer. Ce questionnement s'est décliné dans l'ensemble des ateliers sous la forme de deux sous-interrogations :

- comment favoriser la répartition spatio-temporelle d'une ressource alimentaire saine, de qualité et en quantité suffisante au sein des exploitations agricoles pour maintenir une production apicole durable?

- comment limiter les risques d'exposition des insectes pollinisateurs à des substances toxiques issues des activités agricoles? 
Tableau 1. Synthèse de leviers discutés lors des différents ateliers de concertation, pouvant être mis en place au sein d'exploitations de grandes cultures, pour maintenir durablement des activités de grands cultures et apicoles sur un territoire.

\begin{tabular}{lll}
\hline & $\begin{array}{l}\text { Levier favorisant la contribution des systèmes agricoles } \\
\text { à l'alimentation pollinique et nectarifère } \\
\text { des insectes pollinisateurs }\end{array}$ & $\begin{array}{l}\text { Leviers favorisant la gestion du risque d'exposition } \\
\text { des abeilles à des substances toxiques issues de } \\
\text { l'activité agricole }\end{array}$ \\
\hline $\begin{array}{l}\text { À l'échelle } \\
\text { parcellaire }\end{array}$ & $\begin{array}{l}\text { Implantation de cultures de rente fournissant } \\
\text { du pollen et/ou du nectar } \\
\text { Implantation d'un mélange d'espèces mellifères } \\
\text { en période d'interculture (adaptation de la gestion } \\
\text { de ce couvert pour obtenir une floraison précoce, } \\
\text { de juillet à octobre) }\end{array}$ & $\begin{array}{l}\text { Adoption de techniques alternatives et de stratégies } \\
\text { agronomiques pour limiter voire éviter les risques } \\
\text { de dégâts causés par des ravageurs et ainsi limiter } \\
\text { le recourt à des traitements chimiques (densité et date } \\
\text { de semis, associations d'espèces, mélanges variétaux...) }\end{array}$ \\
& $\begin{array}{l}\text { Limiter les risques d'exposition des abeilles aux produits } \\
\text { en période de butinage en traitant de nuit }\end{array}$ \\
En dehors de l'espace \\
de production
\end{tabular}

Les pistes d'actions envisagées au cours des différents ateliers ont été argumentées, parfois critiquées et contextualisées aux problématiques et enjeux locaux.

La mise en situation et les supports de jeu permettent de surmonter les différences d'échelles auxquelles raisonne chaque producteur (rayon de butinage d'un rucher versus parcellaire) et ainsi proposer des évolutions non seulement à l'échelle de la parcelle mais également à l'échelle du système de culture voire du paysage (Tab. 1). Tous les acteurs présents reconnaissent que les leviers évoqués peuvent représenter des atouts et bénéfices pour le système de culture. Cependant, ils s'accordent également à dire que l'adoption massive de certains d'entre eux est dépendante de nombreux autres facteurs. La notion de risque, souvent propre à chaque exploitant a ainsi souvent été abordée. Cette dernière peut être d'ordre technique (manque de références ou d'accompagnement technique...) ou d'ordre économique (manque de débouché, manque de référence sur la marge associée à la pratique, crainte d'un non-retour sur investissement...). Ainsi, bien que les ateliers facilitent les échanges entre professionnels apicoles et agricoles et permettent de faire émerger des pistes d'actions en faveur des insectes pollinisateurs, ils montrent des limitent dans l'appropriation par les participants de ces leviers pour aller jusqu'à leur mise en application.

\section{Accompagner un réseau d'exploitants volontaires pour tester et évaluer en plein champs la durabilité de systèmes de cultures a priori favorables aux insectes pollinisateurs : projet DEPHY-abeille}

L'ITSAP-Institut de l'abeille à travers le projet DEPHYAbeille (ECOPHYTO DEPHY EXPE 2013-2018) souhaite dépasser l'étape de concertation et guider les exploitants à concrétiser les enseignements des ateliers au sein de leur exploitation. Ce projet a pour objectif de mettre à l'épreuve chez des agriculteurs de la zone atelier Plaine et Val de Sèvre (Poitou-Charentes, coordination CEBC-CNRS), des systèmes de cultures innovants a priori favorables aux abeilles, économes en produits phytosanitaires et économiquement viables. Après avoir participé à des ateliers de concertations en présence d'apiculteurs de leurs territoire, 9 exploitants de la plaine de Niort se sont portés volontaires pour expérimenter sur une partie de leur parcellaire, la prise en compte des insectes pollinisateurs dans leurs pratiques. Un collectif d'acteurs du développement (chambre d'agriculture) et de la recherche en écologie, apidologie et agronomie (INRA, CEBC CNRS) accompagne ces exploitants dans la démarche et participe à l'évaluation des changements de pratiques mis en place vis-à-vis de la durabilité économique du système pour l'exploitant agricole et de son attractivité pour les insectes pollinisateurs. Cette expérimentation a débuté à l'automne 2014 pour une durée de 4 ans. Ce paragraphe apporte une réflexion sur les bénéfices de la démarche mise en œuvre dans l'appropriation de la prise en compte des insectes pollinisateurs par les exploitants engagés.

\subsection{Un dispositif pour expérimenter la prise en compte des insectes pollinisateurs en systèmes céréalier en condition d'exploitation}

Neuf exploitants se sont engagés dans le dispositif sur le volontariat avec chacun des motivations propres (baisse d'utilisation d'intrant, faire évoluer certaines pratiques suites aux échanges lors des ateliers de mise en situation, intérêt pour le miel...). Vingt-sept parcelles (3 par exploitant) sont ainsi engagées dans l'expérimentation avec des systèmes de référence très diversifiés (les préparations de semi-allant du labour occasionnel à des semi-directs sous couvert, les successions culturales allant de 3 à 7 ans avec pour têtes de rotation majoritairement du blé, du colza et du tournesol). Au sein de cette pluralité de systèmes, il est proposé aux exploitants d'appliquer sur une partie expérimentale $(\sim 2$ ha par parcelle) des changements techniques pour aller vers des systèmes de cultures économiquement viables, favorables aux insectes pollinisateurs et à l'apiculture. La traduction de cet enjeu en leviers à tester, a reposé à la fois sur des hypothèses 
émises par le collectif, l'appropriation de la thématique par les exploitants du réseau et des connaissances scientifiques (Encadré 1). Les objectifs du dispositif ont ainsi été collectivement définis :

- augmenter la présence de ressource alimentaire pour les insectes pollinisateurs à des périodes stratégiques de faible ressource florale disponible dans le paysage: le mois de juin et la période de pré-hivernale d'automne à travers deux leviers :

- favoriser une ressource inhérente aux parcelles que sont les adventices messicoles par des réductions d'herbicides (essentiellement anti-dicotylédones) et d'azote afin de favoriser un certain type de flore et de jouer sur la compétitivité culture/adventices,

- implanter un mélange d'espèces mellifères en période d'interculture en visant une floraison dès les mois d'août/ septembre;

- diminuer le risque d'exposition des insectes pollinisateurs à des substances toxiques par des impasses, le choix des matières actives utilisées ainsi que des modes et périodes d'applications qui se déclinent en actions concrètes: pas d'utilisation de semences de céréales enrobées par de l'imidaclopride, application des interventions phytosanitaires le soir en saison apicole...

Différents suivis et observations sont par ailleurs menés pour évaluer si les évolutions de pratiques mises en œuvre rendent compatibles :

- la présence de ressource pour les insectes pollinisateurs?

- le revenus de l'agriculteur?

- la réduction de l'usage des pesticides (IFT) global?

\subsection{La force d'un réseau dans l'appropriation de la problématique et la concrétisation d'actions sur le terrain}

Après deux années d'expérimentation, l'ensemble des exploitants ont opéré des changements de pratiques sur les 27 parcelles engagées dans le réseau, de nature et de degrés d'intensité très hétérogènes, en raison notamment de la liberté dont ils disposent dans la mise en application des différents leviers proposés. Nous ne présenterons pas ici les résultats des travaux expérimentaux, toujours en cours mais illustrerons comment l'acquisition de références et la force des échanges collectifs, accompagnent les exploitants du réseau à s'approprier durablement la problématique.

\subsubsection{L'apport de connaissance, une étape clé dans l'évolution du point de vue}

$\mathrm{Au}$ sein du dispositif DEPHY-Abeille, l'attractivité des parcelles pour les insectes pollinisateurs est évaluée à travers des relevés du nombre d'abeilles domestiques, d'abeilles sauvages, de bourdons, de syrphes ou de papillons en action de butinage le long de transects $(50 * 2 \mathrm{~m})$. Ces transects sont réalisés soit sur des cultures potentiellement attractives en différents points de la parcelle, soit sur des patchs de flores
Encadré 1. Connaissances sur le contexte apicole de la zone d'étude du projet DEPHY-Abeille, explicitées auprès des exploitants pour alimenter leur réflexion :

Le territoire d'étude du projet DEPHY-Abeille est la zone atelier Plaine et Val de Sèvre, un territoire animé par le CNRS-CEBC de Chizé, véritable plate-forme de recherche sur les agro-écosystèmes où y sont étudiés la biodiversité et les pratiques agricoles en plaine céréalière. Le dispositif ECOBEE (observatoire de la dynamique de colonies d'abeilles domestiques en plaine céréalière a été créé en 2008 (Odoux et al., 2014) en est un exemple.

$\mathrm{Au}$ sein de cette plaine céréalière, le régime alimentaire des abeilles domestiques est ponctué par les floraisons des cultures oléagineuses de colza et de tournesol qui représentent les apports en nectar majoritaires pour la colonie pendant l'année. Entre ces deux floraisons, les abeilles domestiques connaissent une pénurie florale susceptible de contribuer à leur déclin (Requier et al., 2016). Les colonies pour lesquelles cette période de disette est la moins prononcée, s'approvisionnent en pollen auprès de la flore herbacée comme le coquelicot qui peut représenter jusqu'à $60 \%$ de son bol alimentaire pollinique au mois de juin (Requier et al., 2015).

La période de mise en hivernage pour les colonies d'abeilles qui suit la floraison du tournesol sur ce territoire est également une période stratégique pour la colonie. Cette dernière élève alors les abeilles dont le rôle sera de maintenir la colonie en vie pendant l'hiver. Assurer une alimentation pollinique de qualité pour des larves qui naissent à l'automne est donc vital pour la colonie. Les systèmes agricoles peuvent alors participer à diversifier le régime de l'abeille à cette période par l'implantation de couverts mellifères en période d'interculture (Allier et al., 2017). La physiologie des abeilles (à savoir la masse corporelle et le taux de vitellogénine, une protéine essentielle qui serait impliqué dans la longévité des abeilles d'hiver) peut être significativement améliorée par la présence de couverts d'intercultures mellifères à proximité des ruchers (Alaux et al., 2017). Cependant ces pollen peuvent potentiellement être contaminés par des substances d'origine agricoles comme l'imidaclopride (molécule insecticide systémique employée en enrobage de semences de céréales, rémanente dans le sol, fortement toxique pour les abeilles) (Allier et al., 2017). L'implantation d'espèces potentiellement attractives pour des insectes pollinisateurs, doit intégrer une réflexion sur les risques potentiels de contamination de cette ressource: intervention sur ou à proximité de cette ressource, rémanence de molécules dans les sols pouvant être remobilisés par cette ressource (Botias et al., 2015; Henry et al., 2015).

spontanées ayant levés au sein de parcelles de céréales, sur toute la durée de floraison de ces espèces (Tab. 2).

Ces données permettent de se rendre compte du comportement de la fréquentation des différentes espèces de plantes cultivées ou d'adventices (Fig. 6). Sur le colza et le tournesol, des 
Tableau 2. Caractéristiques des notations réalisées en 2016 sur quelques parcelles expérimentales pour caractériser la fréquentation de ces dernières par des insectes pollinisateurs.

\begin{tabular}{|c|c|c|c|c|c|c|}
\hline & & \multicolumn{4}{|c|}{ Cultures attractives } & $\frac{\text { Adventices }}{\text { Bleuet }}$ \\
\hline & $\begin{array}{l}\text { Nombre de parcelles } \\
\text { suivies }\end{array}$ & 3 & 3 & 1 & 1 & 1 \\
\hline \multirow[t]{2}{*}{ Efforts de mesure } & $\begin{array}{l}\text { Nombre de transects } \\
\text { réalisés }\end{array}$ & 174 & 64 & 18 & 36 & 21 \\
\hline & Période des relevés & $\begin{array}{l}\text { Du } 01 / 04 \\
\text { au } 02 / 05\end{array}$ & $\begin{array}{l}\text { Du } 20 / 07 \\
\text { au } 01 / 08\end{array}$ & $\begin{array}{l}\text { Du } 19 / 05 \\
\text { au } 31 / 05\end{array}$ & $\begin{array}{l}\text { Du } 25 / 04 \\
\text { au } 19 / 05\end{array}$ & $\begin{array}{l}\text { Du } 07 / 06 \\
\text { au } 24 / 06\end{array}$ \\
\hline \multirow{4}{*}{$\begin{array}{l}\text { Nombre d'insectes } \\
\text { pollinisateurs observés }\end{array}$} & Abeilles domestiques & 992 & 745 & 115 & 374 & 97 \\
\hline & Abeilles sauvages & 87 & 35 & 13 & 34 & 51 \\
\hline & Bourdons & 82 & 115 & 24 & 81 & 91 \\
\hline & Papillons & 14 & 5 & 1 & 3 & 4 \\
\hline
\end{tabular}

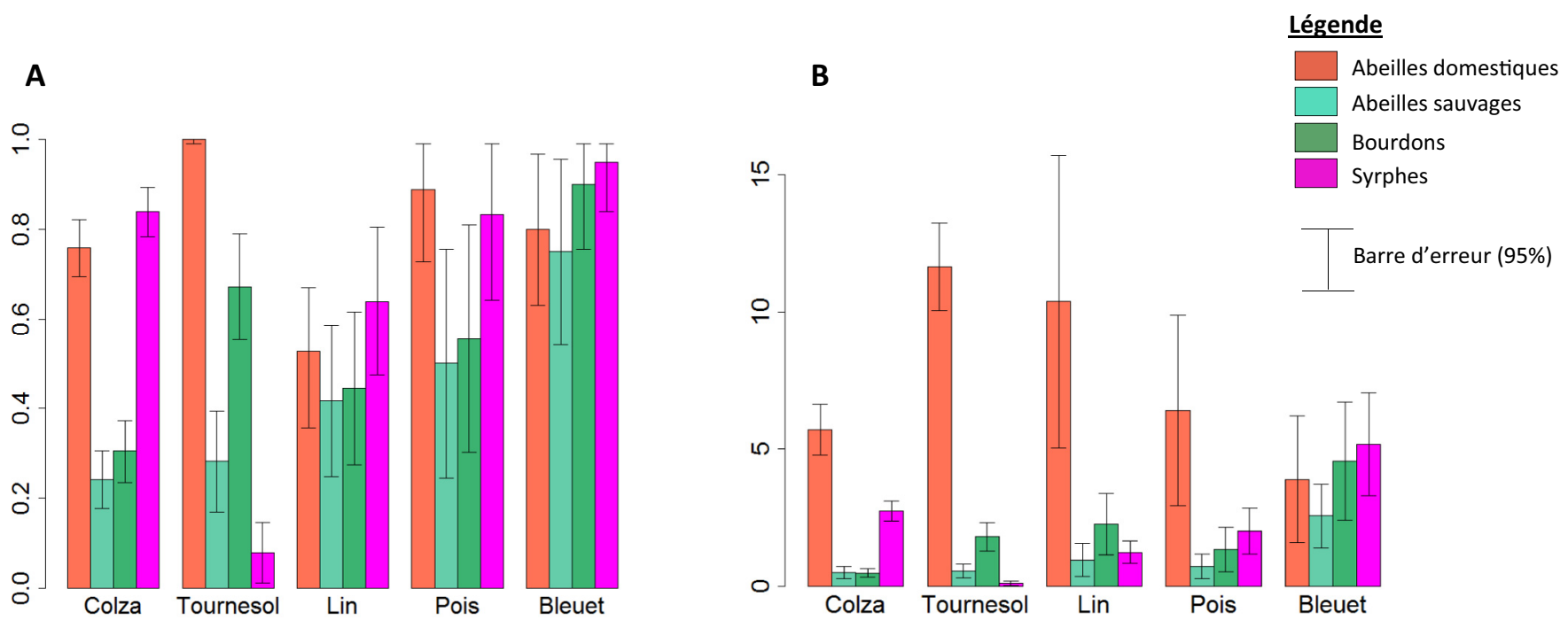

Fig. 6. Fréquence d'occurrence (A) et abondance (B) des différents groupes d'insectes pollinisateurs pour différents habitat (des cultures de rente: colza, tournesol, lin, pois ou des patchs de flore spontanée ayant émergée dans des parcelles de blé : bleuet).

références similaires avaient été données sur ce même territoire en 2013 (Rollin et al., 2013). L'extension des mesures à d'autres cultures et aux adventices, enrichit les connaissances sur les espaces agricoles fréquentés par ces insectes pollinisateurs en plaine agricole mais est également un élément qui alimente les réflexions des exploitants sur la perception de leurs espaces de productions. Les espèces cultivées ne sont plus seulement productrices de grains et certains adventices ne sont plus seulement perçues comme des nuisibles pour la production, ce sont également des ressources potentielles en nectar et pollen pour les insectes pollinisateurs. Par ailleurs, les abeilles domestiques ne sont pas les seuls insectes polinisateurs à fréquenter les parcelles cultivées. Même si parfois en moyenne le nombre de pollinisateurs observés lors d'un relevé n'est pas très élevé (exemple entre 1 et 2 syrphes en moyenne observé lors d'un transect sur pois, Fig. 6B), la probabilité d'en observer au moins un lors d'un relevé est très élevé ( $>80 \%$ pour les syrphes sur pois, Fig. 6A). Des cultures non productrices de miel peuvent donc être des ressources convoitées tout au long de leur floraison par des insectes pollinisateurs. L'acquisition de ce type de références au sein d'un réseau de producteurs permet à ces derniers de s'approprier les résultats : le colza et le tournesol (cultures réputées dans le secteur pour permettre une production de miel) ne sont plus à leurs yeux les seuls espaces agricoles fréquentés par des insectes pollinisateurs. Ces collectes de données sont donc importantes pour amener les exploitants à se questionner sur les différents rôles que peuvent jouer leur parcellaire pour les insectes pollinisateurs.

\subsubsection{Les échanges en réseau, une étape vers la concrétisation d'actions}

En plus d'acquérir de nouvelles connaissances, l'expérimentation en réseau permet d'échanger collectivement sur les observations et mesures issues de l'expérimentation. Ces temps de discussions sont l'occasion d'aborder les difficultés 
rencontrées, faire un constat commun sur de nouvelles références acquises et même proposer collectivement des actions concrètes à mener. Ainsi, bien que certains exploitants n'envisagent pas à cette étape de l'expérimentation de reproduire les changements opérés sur l'ensemble de leur parcellaire, l'ensemble des exploitants impliqués dans le dispositif manifeste un intérêt grandissant pour les insectes pollinisateurs que ce soit par l'adoption de certains leviers sur le reste de leur parcellaire ou bien la mise en œuvre sur d'autres parcelles, de nouveaux leviers non retenus dans le dispositif. La diversification des formats d'échanges et des supports d'information favorisent ainsi l'appropriation par les exploitants des enjeux liés aux insectes pollinisateurs au-delà des parcelles engagées en expérimentation.

\section{Conclusion}

La prise en compte des insectes pollinisateurs comme un objectif à part entière dans la conception et mise en œuvre d'un système de cultures nécessite un changement de paradigme pour l'ensemble des acteurs de la filière agricoles. L'ITSAPInstitut de l'abeille essaie d'accompagner cette évolution en proposant aux acteurs d'un territoire de s'approprier la question pour proposer voire mettre en œuvre des actions pour y répondre. Les différents travaux illustrés dans ce document démontrent l'importance de diversifier les formats d'accompagnement et d'expérimentation afin de sensibiliser le plus grand nombre et de laisser une liberté d'action sur le terrain. Ils soulèvent également la question des catégories d'acteurs à impliquer dans le processus d'évolution des pratiques. Élus, consommateurs, représentants des services de l'état, beaucoup de parties prenantes autres que les producteurs sont concernés par la problématique et pourraient être impliqués dans des démarches similaires afin d'élargir le choix des possibles quant à la prise en compte des insectes pollinisateurs dans l'évolution des territoires agricoles.

\section{Références}

Alaux C, Allier F, Decourtye A, et al. 2017. A 'Landscape physiology' approach for assessing bee health highlights the benefits of floral landscape enrichment and semi-natural habitats. Sci Rep 7: 40568.

Allier F, Alaux C, Aupinel P, et al. 2017. Tester une innovation technique favorable aux abeilles mellifères par des approches participative et expérimentale-Projet InterAPI. Innov Agron 55: $13-28$.
Barreteau O, Bousquet F, Etienne $\mathrm{M}$, Souchère V, D'Aquino $\mathrm{P}$. Chapitre 1-La modélisation d'accompagnement : une méthode de recherche participative et adaptative. In: Etienne M, ed. Quæ, Modélisation d'accompagnement. 2010, pp. 21-46.

Bommarco R, Marini L, Vaissière B. 2012. Insect pollination enhances seed yield, quality, and market value in oilseed rape. Oecologia 169(4): 1025-1032.

Botias C, David A, Horwood J, et al. 2015. Neonicotinoid residues in wildflowers, a potential route of chronic exposure for bees. Environ Sci Techno 49(21): 12731-12740.

Cressey D. 2013. Europe debates risk to bees. Nature 496: 408.

Daré W, Ducrot R, Botta A, Etienne M. 2009. Repères méthodologiques pour la mise en œuvre d'une démarche de modélisation d'accompagnement. Laudun: Cardère éditions, $127 \mathrm{p}$.

Etienne M, Du Toit D, Pollard S. 2011. ARDI: a co-construction method for participatory modeling in natural resources management. Ecol Soc 16(1): 44.

Gem-Oniflhor. 2005. Audit de la filière miel 2005.

Henry M, Cerrutti N, Aupinel P, et al. 2015. Reconciling laboratory and field assessments of neonicotinoid toxicity to honeybees. Proc R Soc B 282: 20152110.

Maxim L, Sluijs JVD. 2013. Seed-dressing systemic insecticides and honeybees. The Bayer CropScience view on Maxim and van der Sluijs "Seed- dressing systemic insecticides and honeybees: a challenge for democratic governance of controversies on chemical risks"(1). European Environment Agency, pp. 1-20.

Odoux J, Aupinel P, Gateff S, Requier F, Henry M. 2014. ECOBEE: a tool for long-term bee colony monitoring at landscape scale in West European intensive agroecosystems. $J$ Apic Res 53(1): 57-66.

Potts SG, Biesmeijer JC, Kremen C, Neumann P, Schweiger O, Kunin WE. 2010. Global pollinator declines: Trends, impacts and drivers. Trends Ecol Evol 25(6): 345-353.

Requier F, Odoux J, Tamic T, et al. 2015. Honey bee diet in intensive farmland habitats reveals an unexpectedly high flower richness and major role of weeds. Ecol Appl 25(4): 881-890.

Requier F, Odoux J, Henry M, Bretagnolle V. 2016. The carry-over effects of pollen shortage decrease the survival of honeybee colonies in farmlands. J Appl Ecol 54: 1161-1170.

Rollin O, Bretagnolle V, Decourtye A, et al. 2013. Differences of floral resource use between honey bees and wild bees in an intensive farming system. Agric Ecosyst Environ 179(2013): 78-86.

Stanley DA, Gunning D, Stout JC. 2013. Pollinators and pollination of oilseed rape crops (Brassica napus L.) in Ireland: ecological and economic incentives for pollinator conservation. $J$ Insect Conserv 17: 1181-1189.

Suryanarayanan S, Kleinman DL. 2013. Be(e)coming experts: The controversy over insecticides in the honey bee colony collapse disorder. Soc Stud Sci 43(2): 215-240.

Citation de l'article : Gourrat M. 2017. De la concertation territoriale à l'expérimentation en plein champs, différents leviers pour accompagner les acteurs d'un territoire agricole à façonner des paysages durablement favorables à des productions oléagineuses et des productions de miel. OCL 24(6): D605. 